\title{
Holographic multiplexing in photorefractive polymers
}

\author{
Gregory J. Steckman ${ }^{\mathrm{a}, *}$, Reinhard Bittner ${ }^{\mathrm{b}}$, Klaus Meerholz ${ }^{\mathrm{b}}$, Demetri Psaltis ${ }^{\mathrm{a}}$ \\ a Department of Electrical Engineering, California Institute of Technology, Mail Stop 136-93, Pasadena, CA 91125, USA \\ ${ }^{\mathrm{b}}$ Department of Physical Chemistry, University of Munich, Butenandtstrasse 11, D-81377 Munich, Germany
}

Received 27 July 2000; accepted 23 August 2000

\begin{abstract}
A new multiplexing schedule is derived for multiplexing holograms in photorefractive polymers which do not exhibit mono-exponential recording behavior. An $M$-number $(M / \#)$ of 0.3 was measured experimentally by recording 20 holograms of roughly equal strength in a single location of $125-\mu \mathrm{m}$-thick material using peristrophic multiplexing. The effects of hologram dark-decay on the time-evolution of the $M / \#$ and the relative strengths of individual holograms is investigated. (c) 2000 Elsevier Science B.V. All rights reserved.
\end{abstract}

Keywords: Holography; Photorefractive polymer; Recording schedule; Multiplexing

Holographic data storage is a promising technology for the storage of large amounts of data. In order to be useful for holographic data storage applications, a material must be capable of achieving a high $M / \#$, a property dependent on both the recording and erasure dynamics of the stored holograms. In this letter we report the recording of multiple holograms by peristrophic multiplexing in the novel material class of photorefractive (PR) polymers. A new recording schedule is devised to account for the variation of the erasure time constants as a function of exposure. The M/\# measured from the recording of 20 equalized holograms was 0.3 .

The standard structures are sandwiches of the PR polymer between two glass slides coated with transparent electrodes made from indium tin oxide

\footnotetext{
${ }^{*}$ Corresponding author. Fax: +1-626-568-8437.

E-mail address: steckman@sunoptics.caltech.edu (G.J. Steckman).
}

[1-6]. In particular, we used a composite derived from the first high-performance PR polymer [7], consisting of (by weight) $42 \%$ poly-( $N$-vinylcarbazole) (PVK, polymer host), 7\% $N$-ethylcarbazole (plasticizer), 25\% each of the non-linear chromophores 2,5-dimethyl-4,4'-nitrophenylazoanisole and 3-methoxy-4,4'-nitrophenylazoanisole, and $1 \%$ 2,4,7-trinitrofluorenone (sensitizer). The active layer thickness was $d=125 \mu \mathrm{m}$. The holographic setup consisted of two plane-wave $633 \mathrm{~nm}$ recording beams at angles of $50^{\circ}$ and $70^{\circ}$ from the surface normal. They were both s-polarized and had equal intensities of approximately $500 \mu \mathrm{W} / \mathrm{cm}^{2}$ at the sample surface. A weak $\left(3.5 \mu \mathrm{W} / \mathrm{cm}^{2}\right)$ p-polarized readout beam counter-propagating to the $50^{\circ}$ recording beam was used to perform a phase conjugate readout of the holograms. Under these conditions and at the externally applied field used throughout these investigations $(E=62 \mathrm{~V} / \mu \mathrm{m}$; necessary to induce bulk non-linearity), the logarithmically averaged holographic response time [8] of the material was $45 \mathrm{~s}$ and the internal diffraction 
efficiency $\eta=0.1$. For erasure, a fourth non-Bragg matched beam with normal incidence was used with an intensity of approximately $1 \mathrm{~mW} / \mathrm{cm}^{2}$.

Generally, as a measure for the data-storage capacity the $M / \#$ [9] is calculated according to:

$M / \#=\left(\frac{A_{0}}{\tau_{\mathrm{r}}}\right) \tau_{\mathrm{e}}$,

where $A_{0}$ is the maximum single-hologram grating strength, $\tau_{\mathrm{r}}$ is the recording time constant, and $\tau_{\mathrm{e}}$ is the erasure time constant. This formalism presumes mono-exponential single-hologram recording and erasure dynamics and implies fairly small refractive index modulations for the individual holograms $(\eta<0.1 ; \Delta n \propto \operatorname{sqrt} \eta$ is assumed $)$ [9]. The first assumption, however, is not fulfilled in organic PR materials: the recording and erasure curves are at least bi-exponential [8]. Alternatively, $M / \#$ can be determined experimentally by recording multiple holograms of approximately equal strength and then summing the grating strength of the recorded number of holograms, which is defined as sqrt $\eta$ [10]. The most important issue for the latter approach is to have a suitable exposure schedule which allows to record multiple holograms of nearly equal strength. Therefore, single-hologram erasure curves were measured. An important finding of these experiments was the fact that the erasure dynamics depended on the recording time. More details about this phenomenon will be published elsewhere. For the purpose of this paper, we measured single-hologram erasure curves for different recording times between 2 $\mathrm{s}$ and $50 \mathrm{~min}$. They were each fitted by a series of decaying exponentials. The strength of the $m$ th hologram after being erased for $t_{\mathrm{e}}$ seconds will be:

$$
\begin{aligned}
w_{m} & =w\left(t_{m}, t_{\mathrm{e}}\right) \\
& =A_{\mathrm{e} 1}\left(t_{m}\right) \mathrm{e}^{-t_{\mathrm{e}} / \tau_{\mathrm{e} 1}\left(t_{m}\right)}+A_{\mathrm{e} 2}\left(t_{m}\right) \mathrm{e}^{-t_{\mathrm{e}} / \tau_{\mathrm{e} 2}\left(t_{m}\right)}+\cdots,
\end{aligned}
$$

where $t_{m}$ is the time for which the $m$ th hologram was recorded, and the coefficients, $A_{\mathrm{e} i}$, as well as the time constants, $\tau_{\mathrm{e} i}$, are determined experimentally by fitting the erasure curves. The strength of the last ( $M \mathrm{th}$ ) hologram is $w_{M}=w\left(t_{M}, 0\right)$, i.e., $t_{\mathrm{e}}=0$ is presumed. The recording time required for the second to last hologram, $t_{M-1}$, can then be computed by numerically solving the relation
$w\left(t_{M-1}, t_{M}\right)=w_{M}$, since the $(M-1)$ th hologram will be erased during the recording of the last hologram. In this way, the times necessary for recording all holograms can be numerically obtained, in backwards order, with the relation

$w\left(t_{m^{\prime}} \sum_{m^{\prime}=m+1}^{M} t_{m^{\prime}}\right)=w_{M}$.

When using this method for computing the recording schedule, the time for recording the last hologram must be chosen carefully depending on the dynamics of the material and the total number of holograms to be recorded. In some instances, a solution cannot be found. If for example the last hologram is recorded for too long, the earlier holograms cannot be recorded to sufficient strengths in order to finally yield equal strengths for all holograms. By contrast, if the time for recording the last hologram is chosen too short, a solution will be found, however, the strengths of all the holograms will not be optimal, i.e. the dynamic range of the material is not entirely used. In order to find the recording schedule that would give the strongest holograms possible, a numerical routine for the optimization of $t_{M}$ was used. The longest allowed recording time was the maximum recording time for which experimental values were obtained. In numerically solving Eq. (3) it is necessary to evaluate Eq. (2) at arbitrary values of $t_{m}$. However, the coefficients and erasure time constants are only known at those discrete values of $t_{m}$ for which experimental measurements were conducted. To circumvent this limitation the measured data were interpolated for those values of $t_{m}$ that did not correspond to a directly measured value.

Using this method, a schedule for recording 11 holograms was computed, but only the last 10 recording times were used to multiplex 10 holograms, since the first computed recording time was unreasonably long $(\gg 1 \mathrm{~h})$. The diffraction efficiencies are shown in Fig. 1 (solid symbols), where it can be seen that although the schedule works in principle, the last few holograms are decreasing in strength. One suspected reason for this is the dark decay that occurs in these materials, but was not taken into account when deriving the recording schedule. Because of the delay in reading out the 


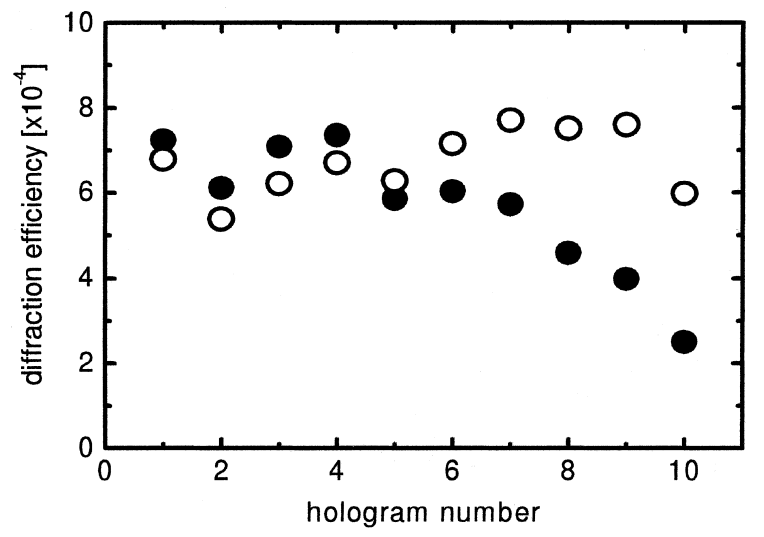

Fig. 1. Diffraction efficiency of 10 multiplexed holograms recorded in the non-doped material using the novel expanded exposure schedule for multiexponential dynamical behavior (๑) and with the empirically altered schedule (plus $1 \mathrm{~s}$ each, $\bigcirc$ ).

holograms, about $0.5 \mathrm{~s}$ each, the later holograms experience stronger decay than the holograms read-out earlier. To compensate for this, the recording schedule was empirically modified by adding $1 \mathrm{~s}$ to each recording time, resulting in more even holograms with an average diffraction efficiency of $6-7 \times 10^{-4}$ (Fig. 1, open symbols). The M/\#s measured for the two schedules by summing the square root of the individual diffraction efficiencies were 0.24 and 0.26 , respectively. Considering the diffraction efficiency of $\eta=0.1$ achieved for a single hologram, which corresponds to a grating strength of 0.32 , it is tempting to utilize the single-hologram diffraction efficiency as an indicator of the possible $M / \#$ for a material. However, this would not be correct since the measurement of $M / \#$ takes into account dynamic effects, such as hologram erasure and dark decay, which are completely ignored in a singlehologram measurement. In other words, a material capable of a large single-hologram index modulation is not necessarily capable of a correspondingly large $M / \#$.

In an attempt to modify the dynamics of the material allowing to record more than 10 holograms, a new composite was created, consisting of the same components used previously, but with the addition of $0.82 \mathrm{wt} \%$ of $N, N^{\prime}$-bis(4-methylphenyl)- $N, N^{\prime}$-bis-(phenyl)-benzidine (TPD), which acts as a hole trap in the PVK photoconducting matrix. This results in reduced charge-carrier mobility in the PR composite and, hence, slower response in both buildup and erasure. Additionally, we expected a reduced dark decay. On the other hand, the steady-state diffraction efficiency for a single hologram was unchanged compared to the material without TPD. The experimental results show that for short recording times subsequent "erasure" actually causes further development of the hologram. The diffraction efficiency reaches a maximum and then begins to decay. This was taken into account by including one growing exponential term into the fits. A detailed study of this interesting effect will be the subject of a forthcoming publication.

Using the same procedure as described above, multiplexing experiments were performed with this modified material to record 20 to 30 holograms (Fig. 2). Overall the uniformity of the recorded holograms is very good with the major deviations existing only at the boundaries. For the case with 20 (30) holograms we measured $M / 0.3(M / 0.23)$. We believe that the $M / \#$ was smaller for the case with 30 than with 20 holograms due to dark decay during the longer time required for read-out. The growth-during-erasure behavior results in exposure schedules whose recordings times are no longer monotonically decreasing with hologram number as in the previous case. Fig. 3 is an illustration of hologram

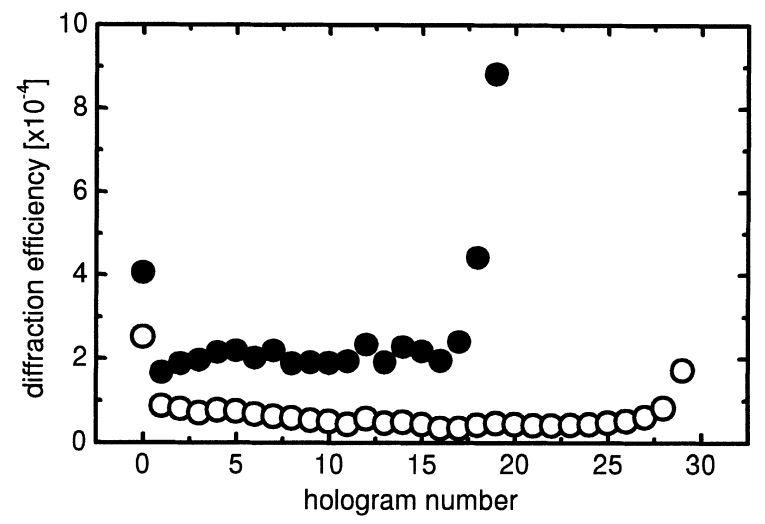

Fig. 2. Diffraction efficiency of multiplexed holograms in the TPD-doped material: 20 holograms $(\mathbf{O}), 30$ holograms $(O)$. 


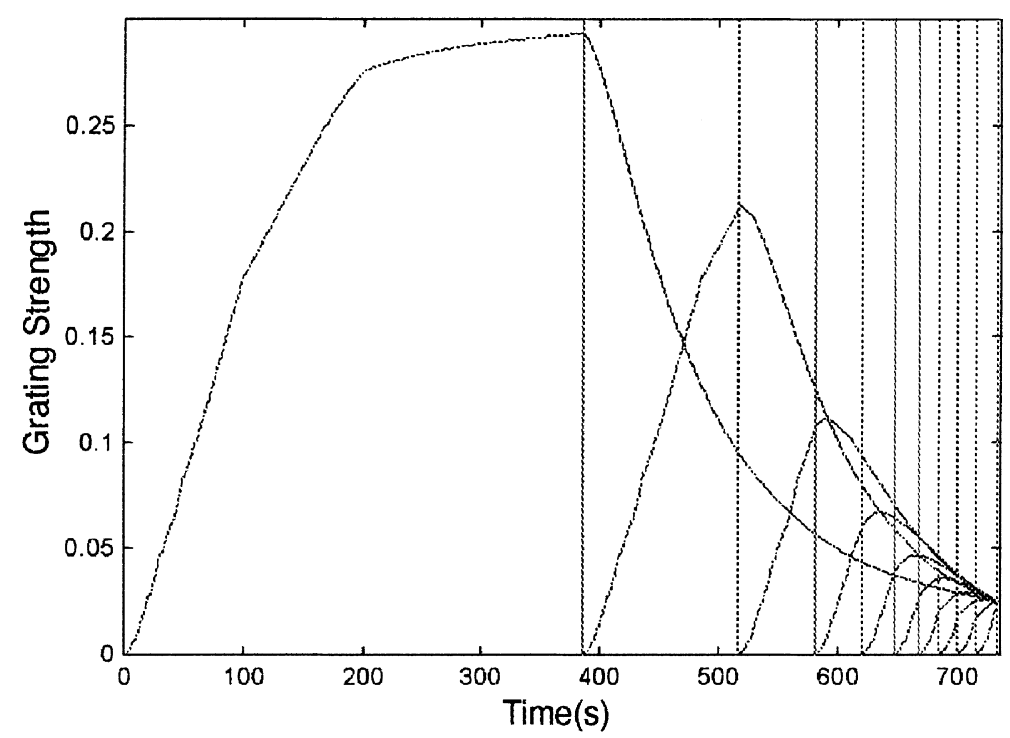

Fig. 3. Illustration of hologram strengths during recording with an exposure schedule for recording 10 holograms in the TPD-doped material. The vertical dashed lines mark the end of recording for one hologram and the start of recording for another.

development during the recording of 10 holograms with this material. The first hologram is recorded to saturation. Due to the erasure time constants increasing with decreasing recording time, subsequent holograms must be recorded beyond the decay of the previous holograms, so that after their erasure they will become equalized. Furthermore, those holograms with shorter recording times experience growth during erasure, allowing in some cases their initial strengths to be less than what they will be at the end of recording. Finally, after the last hologram is recorded, all holograms have either grown or erased so that they have equal strengths.

To get a more detailed insight into the influence of dark decay on the relative strengths of multiplexed holograms in our PR polymer, 20 holograms were recorded with the schedule discussed above and then read out after varying periods of time $t$. Prior to each cycle, gratings remaining from previous recording were erased. As expected, the apparent $M / \#$ decreases with increasing delay $t$ (Fig. 4a), because all holograms decay with time. However, since the decay is different for each individual hologram, the inequality between the holograms increases with $t$ (Fig. 4b), and the cal- culated $M / \#$ loses its significance. These findings also demonstrate that each hologram has a different dark erasure time constant depending on its recording time.

While PR polymers are capable of achieving very large single-hologram diffraction efficiencies, several problems remain to be solved before they

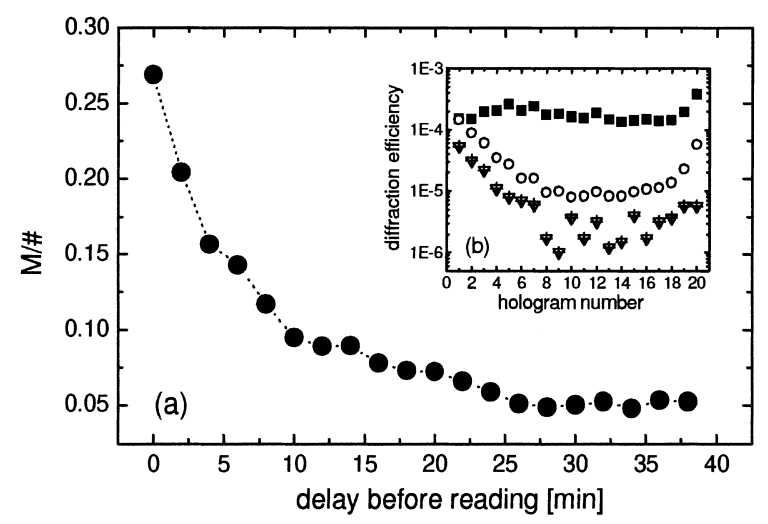

Fig. 4. (a) Dependence of the $M / \#$ on the delay $t$ between recording and the beginning of readout in the TPD-doped material. (b) Diffraction efficiency of 20 multiplexed holograms in the TPD-doped material after delays of $t=0 \min (\boldsymbol{\nabla}), t=10$ $\min (\bigcirc)$, and $t=20 \min (\uplus)$. 
can be applied in holographic data storage. The dependence of the erasure time constant on the initial hologram strength causes the holograms to lose their equality shortly after recording is completed. Furthermore, the fast dark decay times pose serious problems for persistent data storage. However, in its current state the material might be useful in associative memories, where one image is simultaneously compared with all images of a data base, but long-term storage is not intended.

The M/\# of about 0.3 was found to be very similar in both materials. The TPD-doped material allowed for multiplexing of more holograms, which can be attributed to the fact that for short recording times the holograms still increase in strength while being erased. An expanded numerical formalism derived from Ref. [9] and based on multiple exponential buildup and erasure dynamics was developed which allows to determine a suitable recording schedule for an arbitrary storage medium not exhibiting mono-exponential dynamics.

\section{Acknowledgements}

This work was sponsored by DARPA and the Air Force Office of Scientific Research.

\section{References}

[1] W.E. Moerner, S.M. Silence, Chem. Rev. 94 (1994) 127 references therein.

[2] Y. Zhang, R. Burzynski, S. Ghosal, M.K. Casstevens, Adv. Mater. 8 (1996) 111 references therein.

[3] B. Kippelen, K. Meerholz, N. Peyghambarian, in: H.S. Nalva, S. Miyata (Eds.), Nonlinear Optics of Organic Molecules and Polymers, CRC Press, Boca Raton, 1997 (Chapter 8).

[4] K. Meerholz, Angew. Chem. 109 (1997) 981.

[5] K. Meerholz, Angew. Chem. Int. Ed. 109 (1997) 945.

[6] W.E. Moerner, A. Grunnet-Jepsen, C.L. Thompson, Annu. Rev. Mater. Sci. 27 (1997) 585-623.

[7] K. Meerholz, B.L. Volodin, Sandalphon, B. Kippelen, N. Peyghambarin, Nature 371 (1994) 497-500.

[8] R. Bittner, C. Braeuchle, K. Meerholz, Appl. Opt. 37 (14) (1999) 2843-2851.

[9] F.H. Mok, G.W. Burr, D. Psaltis, Opt. Lett. 21 (1996) 896.

[10] A. Pu, K. Curtis, D. Psaltis, Opt. Eng. 35 (1996) 2824. 